\title{
ISOTOPIES VIS-À-VIS LEVEL-PRESERVING EMBEDDINGS
}

\author{
HANSJÖRG GEIGES
}

\begin{abstract}
Various standard texts on differential topology maintain that the level-preserving map defined by the track of an isotopy of embeddings is itself an embedding. This note describes a simple counterexample to this assertion.
\end{abstract}

\section{INTRODUCTION}

Let $Q, M$ be differential manifolds. An embedding of $Q$ in $M$ is a smooth map $\varphi: Q \rightarrow M$ such that $\varphi(Q) \subset M$ is a submanifold and $\varphi$ a diffeomorphism onto its image. Equivalently, an embedding can be characterised as an immersion (i.e. a smooth map of rank equal to $\operatorname{dim} Q$ ) that maps $Q$ homeomorphically onto its image [2, Theorem 1.3.1].

An isotopy of embeddings is a smooth map

$$
F: Q \times[0,1] \longrightarrow M
$$

with the property that for each $t \in[0,1]$ the map

$$
\begin{array}{ccc}
Q & \longrightarrow & M \\
x & \longmapsto & F(x, t)
\end{array}
$$

is an embedding. The track of this isotopy $F$ is the level-preserving map

$$
\begin{aligned}
G: \quad Q \times[0,1] & \longrightarrow M \times[0,1] \\
(x, t) & \longmapsto(F(x, t), t) .
\end{aligned}
$$

It is not difficult to see that any level-preserving embedding

$$
G: Q \times[0,1] \longrightarrow M \times[0,1],
$$

that is, any embedding $G$ satisfying $G(Q \times\{t\}) \subset M \times\{t\}$, is the track of an isotopy [3, Lemma II.4.2]. At least two of the standard texts on differential topology maintain that, conversely, the track of an isotopy is always an embedding [2, p. 178], [3. p. 34]. This claim persists in the literature, at times presented as 'easy to prove'; see [5, p. 164] or [4, p. 207], for instance.

The aim of this brief note is to exhibit a simple counterexample to this assertion; notice that in any such counterexample the manifold $Q$ needs to be non-compact. Thus, we are going to show that the level-preserving map defined by the track of an isotopy of embeddings is not, in general, an embedding.

Remark. This observation is not new, see [1, Aufgabe 9.14], but apparently not nearly as well known as it ought to be.

\section{The EXAMPLe}

In the example we take $Q=\mathbb{R}^{+}$, the positive real numbers, and $M=\mathbb{R}^{2}$.

2010 Mathematics Subject Classification. 57R40, 57R52. 
2.1. A family of bump functions. We first construct, in the standard way, a family of bump functions $h_{t}: \mathbb{R} \rightarrow[0,1]$, where $0<t \leq 1$, with support in the interval $[t / 2,3 t / 2]$. Start with the smooth monotonically increasing function $f$ defined by

$$
f(s):= \begin{cases}0 & \text { for } s \leq 0, \\ \mathrm{e}^{-1 / s} & \text { for } s>0 .\end{cases}
$$

Then the function $g_{t}: \mathbb{R} \rightarrow[0,1]$ defined by

$$
g_{t}(s):=\frac{f(s)}{f(s)+f(t / 4-s)}
$$

interpolates smoothly and monotonically between the value 0 for $s \leq 0$ and the value 1 for $s \geq t / 4$. Finally, set

$$
h_{t}(s):=g_{t}(s-t / 2) \cdot g_{t}(3 t / 2-s) .
$$

This bump function is identically 0 outside the interval $] t / 2,3 t / 2[$, identically 1 on the interval $[3 t / 4,5 t / 4]$, and it interpolates monotonically in between. In particular, we have $h_{t}(t)=1$.

The key to constructing the desired isotopy is the observation that the bump can be made to 'disappear' at $t=0$, provided we restrict the domain of definition to the positive real numbers. In other words, if we take $h_{0} \equiv 0$, then the function $(x, t) \mapsto h_{t}(x)$ will be smooth on $\mathbb{R}^{+} \times[0,1]$.

2.2. The isotopy. We now construct an isotopy $F: \mathbb{R}^{+} \times[0,1] \rightarrow \mathbb{R}^{2}$ of embeddings $\mathbb{R}^{+} \rightarrow \mathbb{R}^{2}$. Set

$$
F(x, t):= \begin{cases}\left(x, h_{t}(x)\right) & \text { for } x \in] 0,2] \text { and } t \in] 0,1], \\ (x, 0) & \text { for } x \in] 0,2] \text { and } t=0, \\ \text { independent of } t, & \\ \text { as shown in Figure } 1 & \text { for } x \geq 2 .\end{cases}
$$

Here it is understood that the choice for $x \geq 2$ is made in such a way that

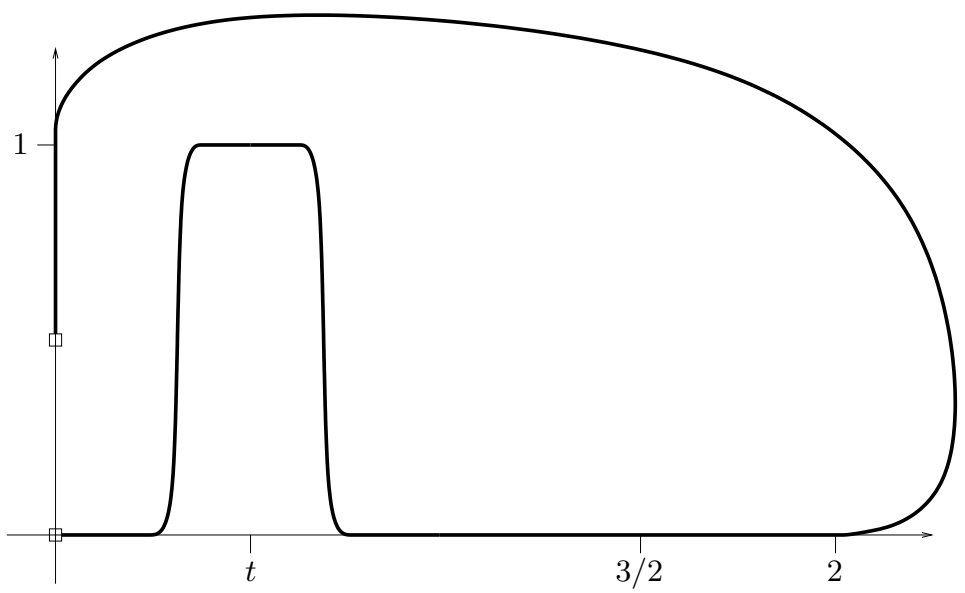

FIgURE 1. The image of the embedding $x \mapsto F(x, t), 0<t \leq 1$. 
$x \mapsto F(x, t)$ is an embedding. The following three points, all of which are a simple consequence of the definition, establish that $F$ is an isotopy.

(i) The map $F$ is smooth at any point $(x, t)$ with $t>0$ or $x>3 / 2$.

(ii) The map $F$ is also smooth at any given point $(x, 0)$ with $0<x \leq 3 / 2$, since for any sequence $\left(x_{\nu}, t_{\nu}\right)$ converging to $(x, 0)$ we have $F\left(x_{\nu}, t_{\nu}\right)=0$ for $\nu$ sufficiently large.

(iii) The map $x \mapsto F(x, t)$ is an embedding $\mathbb{R}^{+} \rightarrow \mathbb{R}^{2}$ for any $t \in[0,1]$.

However, the track $G:(x, t) \mapsto(F(x, t), t)$ of this isotopy is not an embedding, since it is not a homeomorphism of $\mathbb{R}^{+} \times[0,1]$ onto the image of $G$. To see this, choose the unique $x_{0}>2$ such that $F\left(x_{0}, t\right)=(0,1) \in \mathbb{R}^{2}$ for any $t \in[0,1]$. Then $G\left(x_{0}, 0\right)=(0,1,0)$. Now let $\left(x_{\nu}\right)_{\nu \in \mathbb{N}}$ be a sequence of real numbers in $\left.] 0,1\right]$ converging to 0 . Since, by construction, we have $h_{t}(t)=1$ for $0<t \leq 1$,

$$
G\left(x_{\nu}, x_{\nu}\right)=\left(F\left(x_{\nu}, x_{\nu}\right), x_{\nu}\right)=\left(x_{\nu}, 1, x_{\nu}\right),
$$

which converges to $(0,1,0)=G\left(x_{0}, 0\right)$. But the sequence $\left(x_{\nu}, x_{\nu}\right)$ in $\mathbb{R}^{+} \times[0,1]$ does not converge to $\left(x_{0}, 0\right)$.

Remark. (1) The crucial feature of this example is that the embeddings of $\mathbb{R}^{+}$in $\mathbb{R}^{2}$ given by $x \mapsto F(x, t)$ are not proper for $x$ near 0 . It is this improperness which allows one to make a bump 'disappear' smoothly in finite time. The properness or not for $x$ near $\infty$, on the other hand, is irrelevant.

(2) Another way to describe the essential characteristic of this example is to observe that the map

$$
t \longmapsto\{x \longmapsto F(x, t)\}
$$

from the interval $[0,1]$ into the space of embeddings $\mathbb{R}^{+} \rightarrow \mathbb{R}^{2}$ is not continuous at $t=0$ when the space of smooth maps $\mathbb{R}^{+} \rightarrow \mathbb{R}^{2}$ is equipped with the strong topology in the sense of [2, Chapter 2.1].

The recent text by Wall [6], largely based on notes from the 1960s, avoids the pitfall described in this note by working directly with a notion of diffeotopy of $Q$ in $M$, defined as a level-preserving embedding $Q \times[0,1] \rightarrow M \times[0,1]$. Remark (2) serves to indicate that for non-compact manifolds this is in some sense a more appropriate definition.

Acknowledgements. I thank Peter Albers, François Laudenbach, Manfred Lehn and Thomas Rot for useful conversations and correspondence. This note was prompted by Thomas Rot pointing out a related phenomenon to me that he observed during his ongoing work. A search of the literature then revealed that the erroneous assertion about tracks of isotopies continues to appear in text-books. So the example in this note, which I have been discussing in various courses on differential topology over the past 20 years, is for the record.

\section{REFERENCES}

[1] Th. BröCKer AND K. JÄNICH, Einführung in die Differentialtopologie, Heidelberger Taschenbücher 143, Springer-Verlag, Berlin (1973).

[2] M. W. Hirsch, Differential Topology, Grad. Texts in Math. 33, Springer-Verlag, Berlin (1976).

[3] A. A. Kosinski, Differential Manifolds, Pure Appl. Math. 138, Academic Press, Boston, MA (1993).

[4] A. Mukherjee, Differential Topology, 2nd edition, Birkhäuser Verlag, Basel (2015).

[5] A. R. Shastri, Elements of Differential Topology, CRC Press, Boca Raton, FL (2011). 
[6] C. T. C. Wall, Differential Topology, Cambridge Stud. Adv. Math. 156, Cambridge University Press, Cambridge (2016).

Mathematisches Institut, Universität Zu Köln, Weyertal 86-90, 50931 Köln, GerMANY

E-mail address: geiges@math.uni-koeln.de 